\title{
Proarrhythmic Effects of Electrolyte Imbalance in Virtual Human Atrial and Ventricular Cardiomyocytes*
}

\author{
Jermiah J. Joseph, Christopher W. McIntyre, Sanjay R. Kharche* IEEE Member.
}

\begin{abstract}
Dialysis is prescribed to renal failure patients as a long-term chronic treatment. Whereas dialysis therapeutically normalizes serum electrolytes and removes small toxin molecules, it fails to alleviate fibroblast induced structural fibrosis, and unresponsive uremia. The simultaneous presence of altered electrolytes and fibrosis or uremia is thought to be proarrhythmogenic. This study explored potential arrhythmogenesis under pre-dialysis (high electrolyte levels) and post-dialysis (low physiological electrolyte levels) in the presence of fibrosis and uremia in human atrial and ventricular model cardiomyocytes.

Two validated human cardiomyocyte models were used in this study that permitted simulation of cardiac atrial and ventricular detailed electrophysiology. Pathological conditions simulating active fibrosis and uremia were implemented in both models. Pre- and post-dialysis conditions were simulated using high and low electrolyte levels respectively. Arrythmogenesis was quantified by computing restitution curves that permitted identification action potential duration and calcium transient alternans instabilities.
\end{abstract}

In comparison to control conditions, fibrosis abbreviated action potential durations while uremia prolonged the same. Under pre-dialysis conditions, an elevation of serum electrolyte levels caused action potential durations to be abbreviated under both fibrosis and uremia. Alternans instability was observed in the ventricular cardiomyocyte model. Under post-dialysis conditions, lower levels of serum electrolytes promoted an abbreviated action potential duration under fibrosis but caused a large increase of the control and uremic action potential durations. Alternans instabilities were observed in the atrial cardiomyocyte model under post-dialysis conditions at physiological heart rates. The calcium transient restitution showed similar alternans instabilities.

Co-existing conditions such as fibrosis and uremia in the presence of unphysiological electrolyte levels promote arrhythmogenesis and may require additional treatment to improve dialysis outcomes.

Clinical Relevance. Knowledge of model response to clinically relevant conditions permits use of in silico modeling to better understand and dissect underlying arrhythmia mechanisms.

\section{INTRODUCTION}

Hemodialysis is a life prolonging treatment that normalizes serum electrolyte levels (extracellular ionic concentrations) and remove soluble toxins of small molecular size from renal failure patients' blood. However, dialysis is proarrhythmic in nature as a result of multiple factors $[1,2]$. There is significant

*Research supported by funding from the Kidney Clinical Research Unit, Lawson Health Research Institute, London Ontario, Canada.

JJJ is a fourth year undergraduate student in Integrated Science with an Honors Specialization in Computer Science, Western University.

CWM is a practicing clinical nephrologist with London Health Science Centre, ON, Canada and Professor of Medicine and Medical Biophysics at Western University.
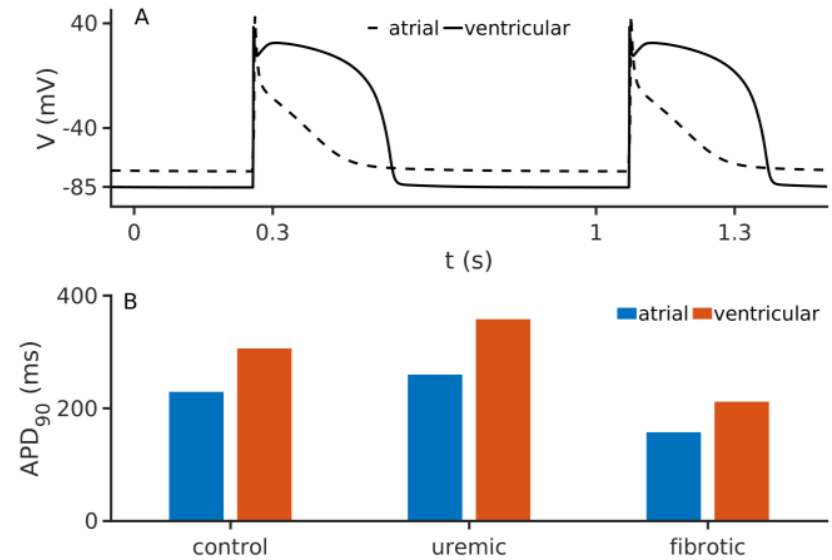

Figure 1. Baseline atrial and ventricular action potentials. A: Action potentials produced by the atrial (dashed line) and ventricular (solid line) cardiomyocyte models. B: Action potential durations $\left(\mathrm{APD}_{90}\right)$ for both cell models (atrial in blue, ventricular in red) under control, uremic, and fibrotic conditions.

accumulation of serum potassium, sodium, and calcium during the intra-dialectic interval. The accumulated levels, or their rapid removal may both be arrhythmogenic [3, 4]. Renal failure confounds the condition by promoting irreversible conditions such as cardiac fibrosis [5]. Active fibrosis is known to affect both organ level conduction $[6,7]$ as well as cardiomyocyte electro-mechanical function $[7,8]$ and reduces the ECG's QT interval. Whereas dialysis is capable of removing a wide range of toxins, certain uremic molecules are irresponsive to uremia [9] leading to prolonged QT intervals. Simultaneous to fibrosis and uremia, renal failure patients often experience cardiac ectopy [10] that initiates fibrillation episodes due to instabilities such as alternans [11, 12]. The effects of the combination of electrolyte imbalance, untreatable fibrosis and uremic conditions, and ectopy on cardiomyocyte electrical and calcium dynamics remains under explored.

Computational cardiac models provide valuable insight regarding electro-dynamic processes that underlies health and disease conditions. We have developed a computational cardiology platform called Precision Medicine using Mathematical Modelling $\left(\mathrm{PM}^{3}\right)$ to interpret data and uncover the processes. Using $\mathrm{PM}^{3}$, this preliminary study aimed to elicit the potentially arrhythmic response of human atrial and ventricular cardiomyocytes to fibrosis and uremia in light of electrolyte imbalance and ectopy.

*SRK is a Research Scientist at London Health Research Institute; and Adjunct Professor (Research) in Medical Biophysics, and Assistant Professor (teaching) in Applied Mathematics, Western University (corresponding author phone: 519-685-8500x56047; fax: 519-685-8072; email: Sanjay.kharche@1hsc.on.ca). 


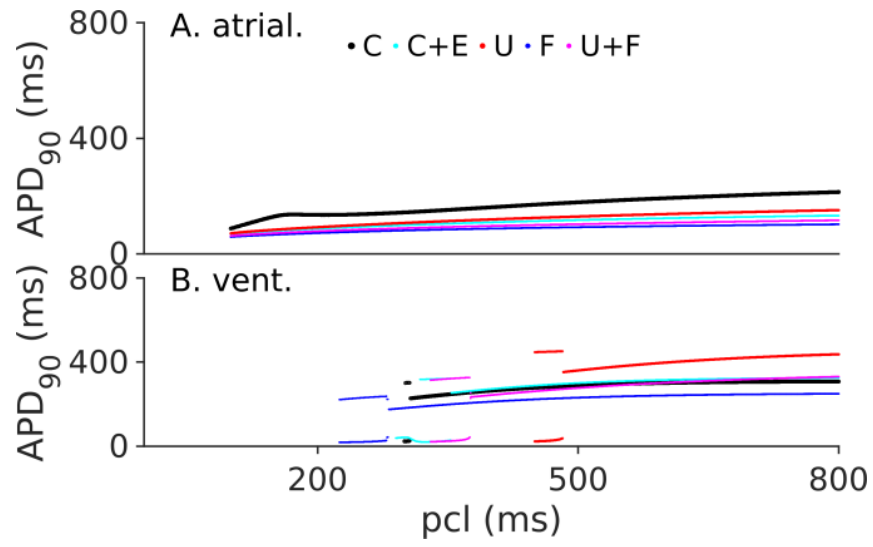

Figure 2. Pre-dialysis action potential duration $\left(\mathrm{APD}_{90}\right)$ restitution in atrial (panel A) and ventricular (panel B) cell models. In both panels, color coding shows control (C, black line), control with increased electrolytes $(\mathrm{C}+\mathrm{E}$, cyan line), uremic (U, red line), fibrotic ( $\mathrm{F}$, blue line), and combined uremic and fibrotic conditions $(\mathrm{U}+\mathrm{F}$, pink line).

Arrhythmogenesis was quantified by simulation of restitution and identification of alternans instabilities in the cardiomyocyte's action potential durations and calcium transient amplitudes.

\section{METHODS}

\section{A. Cell model implementation.}

Two human cardiomyocyte models were obtained from the open source cell model repository [13], CellML, and encoded into our $\mathrm{PM}^{3}$ platform. The cell models are for the human atrial cardiomyocyte by Maleckar et al. [14] and for the human ventricular cardiomyocyte by ten Tusscher et al. $[15,16]$. Action potential profiles and durations of the baseline models are shown in Figure 1.

\section{B. Simulation of electrolyte imbalance, uremia, and fibrosis.}

Electrolyte levels were used to simulate pre- and postdialysis conditions. Under control conditions, both model

cardiomyocytes were simulated using baseline extracellular values (potassium $5.4 \mathrm{mM}$, sodium $140 \mathrm{mM}$, and calcium 1.8 $\mathrm{mM}$ ). Pre-dialysis conditions were simulated using an extracellular potassium value of $10 \mathrm{mM}$, extracellular sodium value of $170 \mathrm{mM}$, and extracellular calcium value of $3 \mathrm{mM}$. Post-dialysis conditions were simulated using extracellular potassium value of $3.2 \mathrm{mM}$, extracellular sodium value of 140 $\mathrm{mM}$, and extracellular calcium value of $1.6 \mathrm{mM}$.

To simulate active fibrosis, electrophysiologically detailed fibroblast cell models were obtained from CellML and encoded into the platform. The atrial fibroblast cell model was coupled to the atrial cardiomyocyte, and the ventricular fibroblast cell model was coupled to the ventricular cell type to simulate atrial or ventricular fibrosis respectively $[7,8]$.

Uremia was simulated by manipulating ionic currents and intra-cellular calcium dynamics. In line with recent experimental data [9], the conductance of the delayed rectifier current was reduced by $50 \%$ and the time constant of L-type

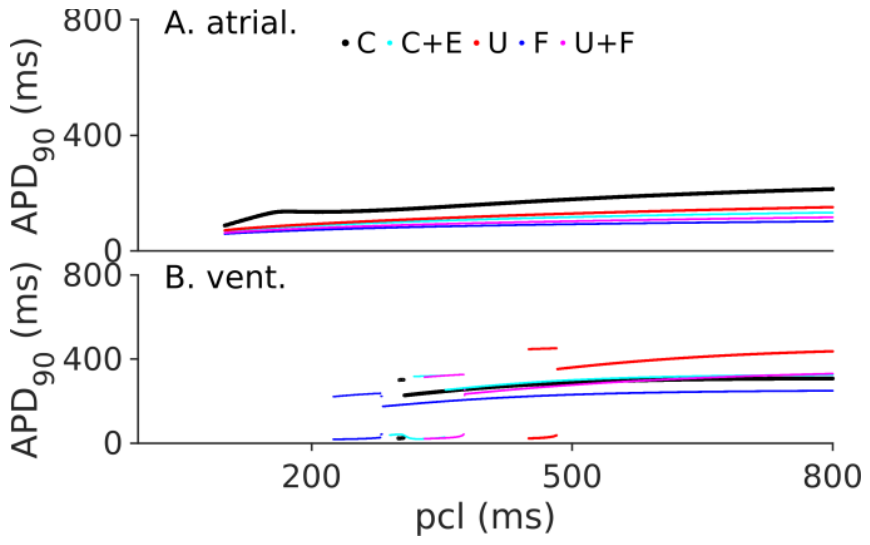

Figure 3. Post-dialysis $\mathrm{APD}_{90}$ restitution for atrial (panel $\mathrm{A}$ ) and ventricular (panel B) cell model under control and pathological conditions. Color coding used is the same as used in Figure 2.

calcium current inactivation slowed (increased) by $60 \%$. Further, the maximal calcium uptake by SERCA into the sarcoplasmic reticulum was reduced by $40 \%$ and calcium release parameter were increased by $50 \%$ in line with existing data [9, 17-19].

Using the above implementations, four cases consisting of control, uremia, fibrosis, and uremia with fibrosis were simulated, each under pre-dialysis (high electrolytes) and post-dialysis (low electrolytes). Under each condition, restitution was computed by pacing the cell models at a given pacing cycle length and action potential durations $\left(\mathrm{APD}_{90}\right)$ and calcium transients of the final 10 excitations recorded.

\section{Numerical methods.}

The model ordinary differential equations were solved using our $O\left(d t^{6}\right)$ accurate implicit method based on backward difference formulae [20]. All models were simulated using a maximum integration time step of $0.1 \mathrm{~ms}$ and relative tolerance of $10^{-3}$ that provided stable and accurate solutions.

\section{RESULTS}

\section{A. Baseline model behaviour.}

The control (baseline) models' behaviour is illustrated in Figure 1. At physiological pacing cycle duration of $814 \mathrm{~ms}$, the atrial cell model had an APD 90 of $229.4 \mathrm{~ms}$ while the ventricular cell model has an APD $_{90}$ of $306.5 \mathrm{~ms}$. Under uremic conditions, the $\mathrm{APD}_{90}$ s were $358 \mathrm{~ms}$ and $260 \mathrm{~ms}$ for the atrial and ventricular cardiomyocyte models respectively. Under fibrosis conditions, the APD 90 's were $157 \mathrm{~ms}$ and 211 $\mathrm{ms}$ for the atrial and ventricular cell models respectively.

\section{B. Incidence of proarrhythmic $A P D_{90}$ alternans before and after dialysis.}

The pre-dialysis $\mathrm{APD}_{90}$ restitution curves are shown in Figure 2. Under pre-dialysis conditions, all diseased atrial $\mathrm{APD}_{90}$ were shorter in comparison to control. Alternans were not observed in the heart rate (i.e. pacing cycle length) range explored. In the ventricular cell model, the uremic APD 90 were longer whereas the fibrotic conditions produced a shorter $\mathrm{APD}_{90}$. In the control case, alternans was observed at very high heartrates with pacing cycle lengths less than $308 \mathrm{~ms}$. In contrast, alternans was observed in the diseased ventricular 

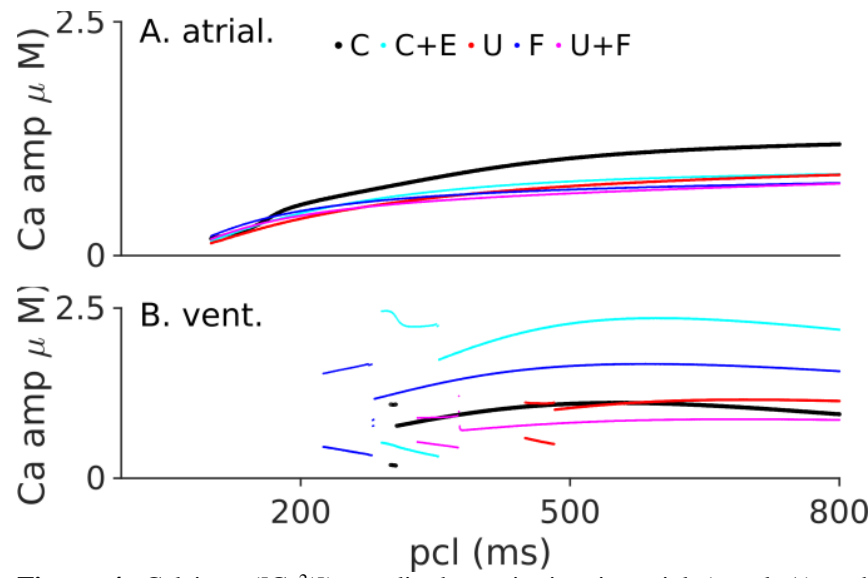

Figure 4. Calcium $\left(\left[\mathrm{Ca}^{2+}\right]\right)$ amplitude restitution in atrial (panel A) and ventricular (panel B) cell models under pre-dialysis conditions in control and pathological cases. Color coding used is the same as in Figure 2

cell models at clinically significant heart rates, i.e. when pacing cycle lengths were less than $490 \mathrm{~ms}$. Uremic conditions promoted alternans at the most clinically significant heart rates while fibrosis suppressed alternans activity (Figure 1, B).

The post-dialysis $\mathrm{APD}_{90}$ restitution curves are shown in Figure 3. Under post-dialysis conditions (low or physiological extracellular electrolyte levels), APD 90 was significantly prolonged due to the altered electrolyte levels and due to uremia in the atrial cardiomyocytes. (Figure 3, A). Uremia caused a large $\mathrm{APD}_{90}$ prolongation in the ventricular cell type, while it was abbreviated due to fibrosis (Figure 3, B). Postdialysis conditions promoted significant alternans under uremic conditions (Figure 3, A) at virtually physiological heart rates in the atrial cell type. Alternans in the atrial cell type devoid of uremia or fibrosis were also observed at physiological heart rates. Under post-dialysis conditions, alternans instabilities in the ventricular cell models under all conditions were observed at relatively higher unphysiological heart rates as compared to the pre-dialysis cases (Figure 3, B).

\section{C. $\mathrm{Ca}^{2+}$ amplitude alternans pre and post dialysis.}

Figures $\mathbf{4}$ and $\mathbf{5}$ illustrate the calcium transient amplitude restitutions pre- and post-dialysis respectively.

Before dialysis, the $\mathrm{Ca}^{2+}$ amplitude was largest in the control case compared to all other cases in the atrial cell. The diseased cases in the atrial cell model had similar $\mathrm{Ca}^{2+}$ transient amplitudes at all heart rates. In the ventricular cell model, $\mathrm{Ca}^{2+}$ instability emerged at $308 \mathrm{~ms}$ in the control case. In the control with altered electrolytes case, the instability emerges at a pacing cycle length of $340 \mathrm{~ms}$. In the uremic case, the instability emerges at a pacing cycle length of 490 $\mathrm{ms}$. In the fibrotic case, $\mathrm{Ca}^{2+}$ instability occurs at much higher heart rates (pacing cycle lengths less than $290 \mathrm{~ms}$ ). In the case with both uremia and fibrosis, the calcium instability emerges at a pacing cycle length of $360 \mathrm{~ms}$.

Under post-dialysis conditions, the atrial control, fibrotic, and uremic combined with fibrotic cases all had similar transient calcium amplitudes and did not have alternans instability. However, the control and uremic cases both experienced calcium instability at physiological heart rates, or pacing cycle lengths of around $650 \mathrm{~ms}$. In the ventricular cell
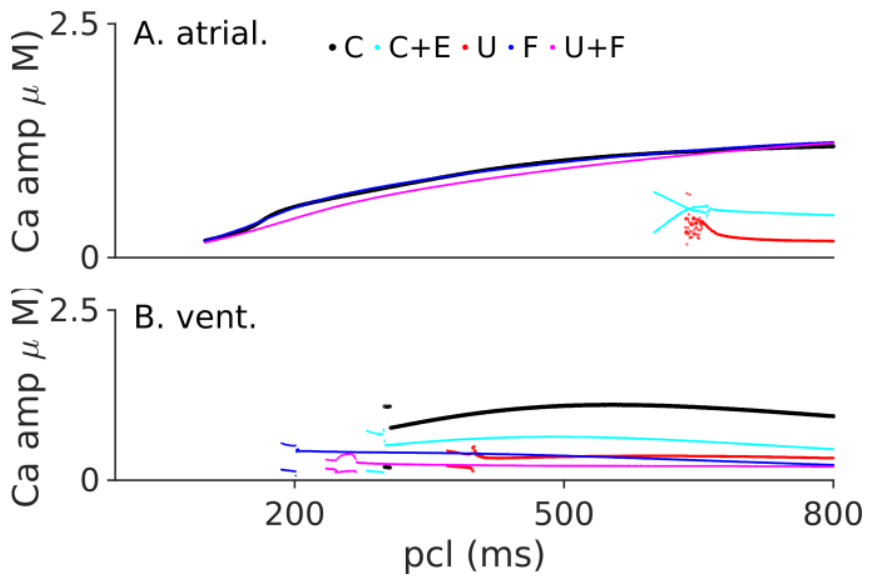

Figure 5. $\left[\mathrm{Ca}^{2+}\right]$ amplitude restitution in atrial (panel A) and ventricular (panel B) cell models under post-dialysis conditions in control and pathological cases. Color coding used is the same as in Figure 2.

types, the control case had calcium transient amplitudes higher than the other cell types. The control case and control with altered electrolytes case both experienced $\mathrm{Ca}^{2+}$ instability at $300 \mathrm{~ms}$. $\mathrm{Ca}^{2+}$ instability emerged in the uremic case around $390 \mathrm{~ms}$. In the fibrotic case, the instability emerged at $200 \mathrm{~ms}$ and at $280 \mathrm{~ms}$ in the uremic combined with fibrotic case.

\section{CONCLUSIONS}

This preliminary study explored the effects of electrolyte imbalance in the presence of uremia or fibrosis pathological conditions on cardiomyocyte electrophysiological activity. From the results, it appears that alternans instability may occur in the ventricles pre-dialysis, while it is certain in the atria postdialysis.

As seen in the restitution curves, fibrosis is an APD90 abbreviating factor. Whereas fibrosis may reduce the cardiomyocytes propensity to sustain alternans, the abbreviated $\mathrm{APD}_{90}$ indicates that single re-entry tachycardia in tissue is likely [21]. Conversely, uremia was found to be an $\mathrm{APD}_{90}$ prolonging condition. Whereas a prolonged $\mathrm{APD}_{90}$ may abrogate tachycardias, the restitution curves show that it promotes functional heterogeneities such as alternans and therefore may give rise to fibrillation at tissue and organ levels [22]. The combined effects of fibrosis and uremia on cardiac action potential are complex and warrant further investigation.

The pathological conditions also had a significant effect on cardiomyocyte calcium transients (Figures 4 and 5). Atrial calcium transients were observed to have lower amplitudes compared to control values under all pathological conditions. The lower amplitude calcium transients potentially promote weaker atrial contractility. In contrast, augmented electrolyte levels and fibrosis were observed to increase calcium transient amplitudes (Figure 4, B), thus indicating hyper-contractility. The mis-match in the atrial and ventricular contractility may promote cardiac dysfunction. Under post-dialysis conditions, both cell types appeared to experience sub-optimal contractility (Figure 5). Alternans instabilities in the calcium transients and $\mathrm{APD}_{90}$ was observed to be linked, which may provide a means to assess contractile function based on noninvasive ECG recording. 


\section{LIMITATIONS AND FUTURE WORK}

This work has simulated diseased conditions using a handful of parameter alterations based on experimental data. However, it is possible that uremia and fibrosis, as well as imbalanced electrolytes affect several other cardiac electrophysiological processes [23, 24]. The cardiomyocyte models considered in this study are limited in their capability to incorporate the effects of important molecules such as extracellular bi-carbonates, phosphates, and $\mathrm{pH}$ [25]. The cell models will be further developed to augment their applicability.

This work explored a specific arrhythmia biomarker in cardiomyocyte models. In future studies, the information obtained from the cell modelling will be extended to multiscale tissue and organ simulations [21] that will provide further insights into disease mechanisms, permitting further classification of the arrhythmia phenotypes.

Variability is an intrinsic feature of biological systems including cardiomyocytes [26]. A number of studies have used variability and variability related methods to elucidate disease mechanisms and tested the potential effectiveness of pharmacological agents [27]. Our past work has introduced novel methods [28] that we will use to further study effects of dialysis on cell and tissue model populations.

\section{ACKNOWLEDGMENT}

We thank Prof. G. Wild (Department of Applied Mathematics, Western University) for his valuable discussions.

\section{REFERENCES}

[1] C. W. McIntyre, "Effects of hemodialysis on cardiac function," Kidney Int, vol. 76, pp. 371-5, Aug 2009.

[2] N. M. Selby and C. W. McIntyre, "The acute cardiac effects of dialysis," Semin Dial, vol. 20, pp. 220-8, May-Jun 2007.

[3] S. Genovesi, C. Dossi, M. R. Vigano, E. Galbiati, F. Prolo, A. Stella, and M. Stramba-Badiale, "Electrolyte concentration during haemodialysis and QT interval prolongation in uraemic patients," Europace, vol. 10, pp. 771-7, Jun 2008

[4] V. P. Yelamanchi, J. Molnar, V. Ranade, and J. C. Somberg, "Influence of electrolyte abnormalities on interlead variability of ventricular repolarization times in 12-lead electrocardiography," Am J Ther, vol. 8, pp. 117-22, Mar-Apr 2001.

[5] M. S. Hulshoff, S. K. Rath, X. Xu, M. Zeisberg, and E. M. Zeisberg, "Causal Connections From Chronic Kidney Disease to Cardiac Fibrosis," Semin Nephrol, vol. 38, pp. 629-636, Nov 2018.

[6] S. R. Kharche, E. Vigmond, I. R. Efimov, and H. Dobrzynski, "Computational assessment of the functional role of sinoatrial node exit pathways in the human heart," PLoS ONE, vol. 12, p. e0183727, 2017.

[7] R. Morgan, M. A. Colman, H. Chubb, G. Seemann, and O. V. Aslanidi, "Slow Conduction in the Border Zones of Patchy Fibrosis Stabilizes the Drivers for Atrial Fibrillation: Insights from Multi-Scale Human Atrial Modeling," Front Physiol, vol. 7, p. 474, 2016.

[8] K. A. MacCannell, H. Bazzazi, L. Chilton, Y. Shibukawa, R. B. Clark, and W. R. Giles, "A mathematical model of electrotonic interactions between ventricular myocytes and fibroblasts," Biophys J, vol. 92, pp. 4121-32, Jun 1 2007.

[9] W. H. Tang, C. P. Wang, F. M. Chung, L. L. Huang, T. H. Yu, W. C. Hung, L. F. Lu, P. Y. Chen, C. H. Luo, K. T. Lee, Y. J. Lee, and W. T. Lai, "Uremic retention solute indoxyl sulfate level is associated with prolonged QTc interval in early CKD patients," PLoS ONE, vol. 10, p. e0119545, 2015. [10] J. O. Burton, S. Korsheed, B. J. Grundy, and C. W. McIntyre, "Hemodialysis-induced left ventricular dysfunction is associated with an increase in ventricular arrhythmias," Ren Fail, vol. 30, pp. 701-9, 2008. [11] L. M. Munoz, A. R. M. Gelzer, F. H. Fenton, W. Qian, W. Lin, R. F. Gilmour, Jr., and N. F. Otani, "Discordant Alternans as a Mechanism for Initiation of Ventricular Fibrillation In Vitro," J Am Heart Assoc, vol. 7, p. e007898, Sep 42018.

[12] K. H. ten Tusscher and A. V. Panfilov, "Alternans and spiral breakup in a human ventricular tissue model," Am J Physiol Heart Circ Physiol, vol. 291, pp. H1088-100, Sep 2006.

[13] C. M. Lloyd, J. R. Lawson, P. J. Hunter, and P. F. Nielsen, "The CellML Model Repository," Bioinformatics, vol. 24, pp. 2122-3, Sep 152008.

[14] M. M. Maleckar, J. L. Greenstein, W. R. Giles, and N. A. Trayanova, "K+ current changes account for the rate dependence of the action potential in the human atrial myocyte," Am J Physiol Heart Circ Physiol, vol. 297, pp. H1398-410, Oct 2009.

[15] K. H. Ten Tusscher and A. V. Panfilov, "Cell model for efficient simulation of wave propagation in human ventricular tissue under normal and pathological conditions," Phys Med Biol, vol. 51, pp. 6141-56, Dec 72006.

[16] K. H. ten Tusscher, D. Noble, P. J. Noble, and A. V. Panfilov, "A model for human ventricular tissue," Am J Physiol Heart Circ Physiol, vol. 286, pp. H1573-89, Apr 2004.

[17] W. T. Chen, Y. C. Chen, M. H. Hsieh, S. Y. Huang, Y. H. Kao, Y. A Chen, Y. K. Lin, S. A. Chen, and Y. J. Chen, "The uremic toxin indoxyl sulfate increases pulmonary vein and atrial arrhythmogenesis," J Cardiovasc Electrophysiol, vol. 26, pp. 203-10, Feb 2015.

[18] A. C. McMahon, S. E. Greenwald, S. M. Dodd, M. J. Hurst, and A. E. Raine, "Prolonged calcium transients and myocardial remodelling in early experimental uraemia," Nephrol Dial Transplant, vol. 17, pp. 759-64, May 2002.

[19] P. Donohoe, A. C. McMahon, O. V. Walgama, F. Bertaso, M. E Dockrell, H. A. Cramp, A. M. Mullen, M. J. Shattock, B. M. Hendry, and A. F. James, "L-type calcium current of isolated rat cardiac myocytes in experimental uraemia," Nephrol Dial Transplant, vol. 15, pp. 791-8, Jun 2000.

[20] A. C. Hindmarsh, P. N. Brown, K. E. Grant, S. L. Lee, R. Serban, D. E. Shumaker, and C. S. Woodward, "SUNDIALS: Suite of Nonlinear and Differential/Algebraic Equation Solvers," ACM Transactions on Mathematical Software, vol. 31, p. 33, 2005.

[21] S. Kharche, C. J. Garratt, M. R. Boyett, S. Inada, A. V. Holden, J. C. Hancox, and H. Zhang, "Atrial proarrhythmia due to increased inward rectifier current (I(K1)) arising from KCNJ2 mutation--a simulation study," Prog Biophys Mol Biol, vol. 98, pp. 186-97, Oct-Nov 2008.

[22] N. Vandersickel, A. Defauw, P. Dawyndt, and A. V. Panfilov, "Global alternans instability and its effect on non-linear wave propagation: dynamical Wenckebach block and self terminating spiral waves," Sci Rep, vol. 6, p 29397, Jul 072016.

[23] E. Grandi, F. S. Pasqualini, C. Pes, C. Corsi, A. Zaza, and S. Severi, "Theoretical investigation of action potential duration dependence on extracellular Ca2+ in human cardiomyocytes," J Mol Cell Cardiol, vol. 46, pp. 332-42, Mar 2009.

[24] S. Severi, E. Grandi, C. Pes, F. Badiali, F. Grandi, and A. Santoro, "Calcium and potassium changes during haemodialysis alter ventricular repolarization duration: in vivo and in silico analysis," Nephrol Dial Transplant, vol. 23, pp. 1378-86, Apr 2008.

[25] A. J. Williams, I. D. Dittmer, A. McArley, and J. Clarke, "High bicarbonate dialysate in haemodialysis patients: effects on acidosis and nutritional status," Nephrol Dial Transplant, vol. 12, pp. 2633-7, Dec 1997. [26] A. X. Sarkar and E. A. Sobie, "Regression analysis for constraining free parameters in electrophysiological models of cardiac cells," PLOS Comput Biol, vol. 6, p. e1000914, 2010.

[27] O. J. Britton, A. Bueno-Orovio, K. Van Ammel, H. R. Lu, R. Towart, D. J. Gallacher, and B. Rodriguez, "Experimentally calibrated population of models predicts and explains intersubject variability in cardiac cellular electrophysiology," Proc Natl Acad Sci U S A, vol. 110, pp. E2098-105, Jun 042013.

[28] S. Kharche, N. Ludtke, S. Panzeri, and H. Zhang, "A Global Sensitivity Index for Biophysically Detailed Cardiac Cell Models: A Computational Approach," LNCS, vol. 5528, p. 10, 2009. 